Check for updates

Cite this: RSC Adv., 2017, 7, 50720

\title{
Tunable solid state emission of novel V-shaped fluorophores by subtle structure modification: polymorphism, mechanofluoro-chromism and micro-fabrication $\dagger$
}

\begin{abstract}
Hong-Yu Fu, Xiao-Jing Liu and Min Xia (D) *
A family of $\mathrm{V}$-shaped fluorophores with different numbers and positions of vinyl are rationally designed, using which a largely wide and approximately successive range of solid-state emission wavelengths can be achieved before and after force stimuli. These compounds are intensely emissive and highly mechanofluorochromic (MFC), and many physical properties such as emission wavelengths, quantum yields, intramolecular charge transfer (ICT) extent, solvatochromism, aggregation-induced emission (AIE) activity and time consumed for restoration from amorphous phase are profoundly influenced by the number and site of vinyl groups. However, the emission wavelength shifts under force stimuli seem to be related to neither the number nor the position of vinyl. For a member with double polymorphs, the unqiue molecular pair packing in one of them revealed by X-ray crystallography is presumably responsible for its MFC inertia and the abnormally one-way phase transformation from the stable to the metastable one. Interestingly, a shape-dependent emission of self-assembled crystal with a fractal structure occurs when both of the two polymorphs are fumed in solvent vapor for long time. Moreover, the films made of these $\mathrm{V}$-shaped compounds are successfully developed to be erasable mediums for information storage with robust fatigue resistance.
\end{abstract}

Received 20th September 2017 Accepted 26th October 2017

DOI: $10.1039 / c 7 r a 10432 d$

rsc.li/rsc-advances

\section{Introduction}

Many materials showing switchable and reversible solid-state luminescence upon exposure to external stimuli have been reported during the last decade. ${ }^{1}$ Among them, mechanofluorochromic ones ${ }^{2}$ produced by small organic molecules are particularly important because of their fundamental relevance and promising applications in sensors, ${ }^{3}$ memory chips, ${ }^{4}$ security inks ${ }^{5}$ or papers, ${ }^{6}$ and optical data storage devices. ${ }^{7}$ Generally, MFC effect occurs on molecules with considerably twisted conformations, ${ }^{8}$ whose loose packing makes corresponding crystals readily collapsed into amorphous phase under force stimuli. Therefore, a red-shifted emission takes place on amorphous sample, in which a more planar conformation is preferred to be adopted.

It has been reported that "†" or X-shaped cruciforms are MFC-active, ${ }^{9}$ as two conjugated arms intersect at a central core to provide highly-twisted conformations. However, such

Department of Chemistry, Zhejiang Sci-Tech University, Hangzhou 310018, P. R. China.E-mail:xiamin@zstu.edu.cn

$\dagger$ Electronic supplementary information (ESI) available: ${ }^{1} \mathrm{H}$ and ${ }^{13} \mathrm{C}$ NMR spectra, MS spectra, absorption and emission spectra, PXRD patterns, DLS diagrams, X-ray crystallography and others. CCDC 1551666 and 1551667. For ESI and crystallographic data in CIF or other electronic format see DOI: 10.1039/c7ra10432d cruciforms always have highly symmetric and complicated skeletons, which lead to the poor solubility of them in common solvents and low crystallization in solid state. ${ }^{10}$ Moreover, mono-functionalized side products are inevitably generated, which results in the tedious work-up and significantly reduced yields of desired double-functionalized cruciforms. Recently, a series of V-shaped molecules, $(E)-2^{\prime}-[2-($ benzo $[d]$ thiazol-2-yl) vinyl]- $N, N$-dimethyl-(1,1'-biphenyl)-4-amine and its analogous, which can be considered as half-cut X-shaped cruciforms, have been developed by our group. ${ }^{11,12}$ They exhibit the reversible MFC activity with vividly contrasted colour change in exposure to force stimuli and render a practical solution to the abovementioned shortcomings of X-shaped cruciforms.

In spite of high MFC activities, the range of solid-state emission wavelengths for ever reported compound family is quite limited and discontinuous before and after force stimuli. In order to realize such continuity, herein a new family with different number of vinyl inserted between dimethylaminophenyl or 2-benzo[d]-thiazolyl and phenyl core at different position is rationally designed as a part of the serial studies on $\mathrm{V}$-shaped fluorophores. It is widely believed that emission color of a molecule is intrinsically dependent on the electronic property of its frontier molecular orbits (FMOs), and even the subtle structure modification certainly has some influence on such property. Hence, the different number and site of vinyl, 
a commonly used $\pi$-electron unit, whose introduction into a $\mathrm{V}$ shaped molecule will vary the length of $\pi$-conjugated region, will finely adjust the FMOs electron property of the corresponding compound. Actually, the produced molecules under such design strategy display the closely structure-related solution-state physical features like emission wavelength, quantum yield, solvatochromism, aggregation-induced emission (AIE) activity. Moreover, as solid-state physical properties are dependent not only on individual molecules but also on their packing modes, making polymorphism and differentiated solid-state physical properties feasibly occur on them. It is found that emission wavelength and time taken for recovery from amorphous to crystalline phase are largely different among compounds in this work. By this subtle structure modification, these molecules successfully exhibit a largely wide and approximately continuous range of solid-state emission wavelengths before and after force stimuli.

Apart from MFC mechanism, polymorphism is another practical method to provide tunable solid-state fluorescence. In general, multicolour polymorphs are several thermodynamically stable or metastable crystals packed by molecules with remarkably different conformations. Meanwhile, their solidstate emission wavelengths together with the powder X-ray diffraction (PXRD) patterns are largely different from each other. ${ }^{13}$ For a polymorph whose conformation is less twisted, it exhibits longer-wavelength emission due to the relatively strengthened $\pi$-conjugation. In this work, the compound $\mathbf{V}_{\mathbf{3}}$ with a vinyl inserted between the dimethyl-aminophenyl and the phenyl core produces two polymorphs, which respectively emit green and yellow fluorescence. However, only the green one is MFC-active, and the transformation between them is just one-way from the yellow to the green one. X-ray diffraction reveals that unique molecular pairs emerge in the yellow one, which is assumed to account for its MFC inertia and the oneway transformation.

Recently, the crystal micro-/nano-fabrication has been an attractive topic in field of functional organic materials, as micro-/nano-structures formed by such means to show specific shapes like wires, rods and ribbons have exhibited their distinguished optical properties. ${ }^{14}$ In contrast to polymorphs, different emissions of such micro-/nano-particles are significantly dependent on size, shape or defects of crystals rather than molecular conformations. Herein, we report that particular micro-crystal of $\mathbf{V}_{\mathbf{3}}$ with fractal structure exhibits orange fluorescence and can be transformed from either the green or the yellow polymorph by a probable dissolution-recrystallization process under prolonged exposure to solvent vapour. It is demonstrated that the PXRD pattern of the orange crystal is the same as that of the green polymorph but thoroughly different from that of the yellow one.

\section{Results and discussions}

\section{Synthesis}

The family of the V-shaped fluorophores have four members and each of them contains a dimethylaminophenyl as the electron-donating group and a 2-benzo[ $d]$ thiazolyl as the
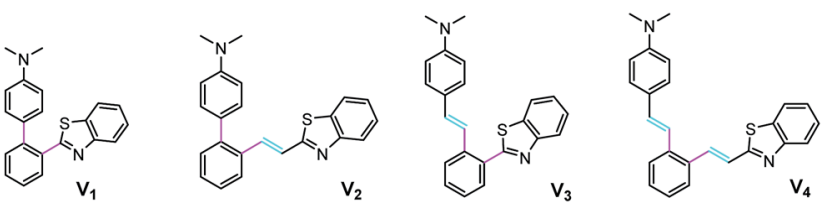

Fig. 1 Chemical structures of $V$-shaped fluorophores $V_{1}-V_{4}$.

electron-withdrawing one (Fig. 1). For them, different number of vinyl is introduced at different position: $\mathbf{V}_{\mathbf{1}}$ with no vinyl, $\mathbf{V}_{\mathbf{2}}$ with one vinyl located between 2-benzo[ $d]$ thiazolyl and phenyl core, $\mathbf{V}_{\mathbf{3}}$ with one vinyl inserted between dimethylaminophenyl and phenyl core, and $\mathbf{V}_{\mathbf{4}}$ with double vinyls.

$4^{\prime}$-(Dimethyl-amino)-[1,1'-bi-phenyl]-2-carbaldehyde, the intermediate 1 , which is obtained by a Suzuki coupling of 2 bromobenzaldehyde and $N, N$-dimethyl-4-(4,4,5,5-tetramethyl1,3,2-dioxaborolan-2-yl)aniline, is respectively carried out a condensation with 2 -aminothiophenol by using DMSO as an oxidant in the air and a Wittig reaction with 2-methylbenzo[d] thiazolyl triphenylphosphonium bromide to produce $\mathbf{V}_{\mathbf{1}}$ and $\mathbf{V}_{\mathbf{2}}$ in moderate to good yields. The similar reactions are also executed to prepare $\mathbf{V}_{\mathbf{3}}$ and $\mathbf{V}_{\mathbf{4}}$ from (E)-2-[4-(dimethylamino) styryl]benzaldehyde, the intermediate 2 , which is gained by a Heck reaction of $\mathrm{N}, \mathrm{N}$-dimethyl-4-vinylaniline and 2-bromobenzaldehyde. All the pristine samples of these compounds are crystals and intensely emissive. The V-shaped fluorophores together with the double intermediates are carefully characterized by ${ }^{1} \mathrm{H},{ }^{13} \mathrm{C}$ NMR and EI-MS, and satisfactory date is obtained to support their structures (ESI $\dagger$ ).

\section{Photophysical property in solution}

Initially, in order to learn about the solution-state emission feature of this family, the emission spectra in $n$-hexane are investigated, because the polarity of $n$-hexane is so small that its solvent effect on the emission wavelengths is very slight. It is shown that the emission wavelengths are respectively located at $443 \mathrm{~nm}$ for $\mathbf{V}_{1}, 459 \mathrm{~nm}$ for $\mathbf{V}_{2}, 460 \mathrm{~nm}$ for $\mathbf{V}_{3}$ and $490 \mathrm{~nm}$ for $\mathbf{V}_{\mathbf{4}}$, which indicates that only the quantity rather than the site of vinyl has influence on them. The more vinyls a V-shaped compound has, the longer emission wavelength it displays. Subsequently, the absorption and emission spectra of this family in different solvents are determined (Fig. S1-4, ESI $\dagger$ ). It is illustrated that the absorption spectra of each member are not solvent-dependent, but the emission ones are very sensitive to the polarity of the solvents. Such sensitivity can be readily observed on the photos of these compounds in different solvents taken under $365 \mathrm{~nm}$ UV light (Fig. 2).

As the solvent polarity rises up, the emission wavelengths make considerably red shifts except $\mathbf{V}_{\mathbf{4}}$ in MeCN (Table 1). It is assumed that the blue shift in MeCN may well be induced by some unknown interactions between $\mathbf{V}_{\mathbf{4}}$ and the solvent. This positive solvatochromism implies that a remarkably enlarged dipole moment accompanying with significant ICT effect occurs on the excited state of each compound. Moreover, the wavelength shift from in $n$-hexane to in acetone is respectively $80 \mathrm{~nm}$ for $\mathbf{V}_{\mathbf{1}}, 119 \mathrm{~nm}$ for $\mathbf{V}_{2}, 88 \mathrm{~nm}$ for $\mathbf{V}_{3}$ and $115 \mathrm{~nm}$ for $\mathbf{V}_{\mathbf{4}}$. 


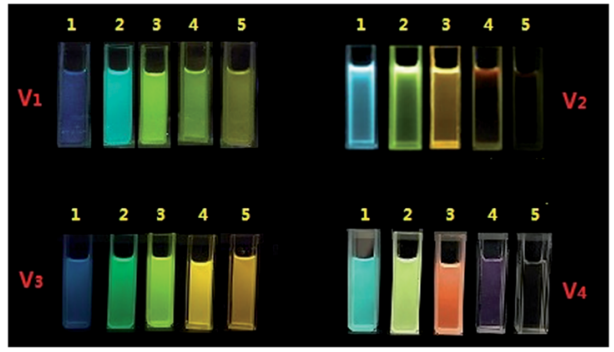

Fig. 2 Photos of $\mathrm{V}_{1}-\mathrm{V}_{4}$ in different solvents under $365 \mathrm{~nm}$ light (1: $n$ hexane; 2: benzene; 3 : dichloromethane; 4: acetone; 5: acetonitrile).

Considering such shift as an indicator to roughly differentiate the extent of ICT effect, it is evidently that the extent is larger in $\mathbf{V}_{\mathbf{2}}$ and $\mathbf{V}_{\mathbf{4}}$ than in $\mathbf{V}_{\mathbf{1}}$ and $\mathbf{V}_{\mathbf{3}}$. Next, the quantum yields of this family in acetone are used as another factor to finely divide the ICT extent. It is measured that they are respectively 0.436 ( \pm 0.025$), 0.113( \pm 0.008), 0.269( \pm 0.017)$ and $0.025( \pm 0.004)$ for $\mathbf{V}_{\mathbf{1}}-\mathbf{V}_{\mathbf{4}}$ (Table 1). Therefore, the extent of ICT effect is in the order of $\mathbf{V}_{\mathbf{1}}<\mathbf{V}_{\mathbf{3}}<\mathbf{V}_{\mathbf{2}}<\mathbf{V}_{\mathbf{4}}$, which means that both the number and the location of vinyl have profound impact on it. A V-shaped compound with the more number of vinyl exhibits the stronger ICT effect, while a fluorophore with a vinyl introduced on the electron-withdrawing group instead of on the electrondonating one produces a heavier ICT effect.

Generally, AIE effect is referred to the phenomenon that solution of a compound is faintly or not emissive, but its aggregation state (including powder or suspension) is intensely emissive. ${ }^{15}$ As the quantum yields of $\mathbf{V}_{\mathbf{2}}$ and $\mathbf{V}_{\mathbf{4}}$ in MeCN are less than 0.01 , water is added as a poor solvent into the MeCN solution of them to check their AIE activity. It is shown (Fig. 3 and S5, ESI $\dagger$ ) that the emission intensity is extremely low when the water fraction in $\mathrm{MeCN} / \mathrm{H}_{2} \mathrm{O}$ mixture is less than $50 \%$, but it is abruptly increased when the water content is respectively enhanced to over $70 \%$ for $\mathbf{V}_{\mathbf{2}}$ and $60 \%$ for $\mathbf{V}_{\mathbf{4}}$. Due to the good hydrophobicity of vinyl, the threshold of water fraction required to produce the emission intensity jump is obviously reduced for $\mathbf{V}_{\mathbf{4}}$. For both $\mathbf{V}_{\mathbf{2}}$ and $\mathbf{V}_{\mathbf{4}}$, the emission intensity is at least 100 -fold higher in $\mathrm{MeCN} / \mathrm{H}_{2} \mathrm{O}(5: 95, \mathrm{v} / \mathrm{v})$ mixture than in pure MeCN solution. The dynamical scattering light (DSL) measurements (Fig. S6, ESI $\dagger$ ) demonstrate that well-dispersed nano-particles with the averaged diameter in $392.1 \mathrm{~nm}$ for $\mathbf{V}_{2}$ and $536.8 \mathrm{~nm}$ for $\mathbf{V}_{\mathbf{4}}$ are formed in $\mathrm{MeCN} / \mathrm{H}_{2} \mathrm{O}(5: 95, \mathrm{v} / \mathrm{v})$ mixture, which

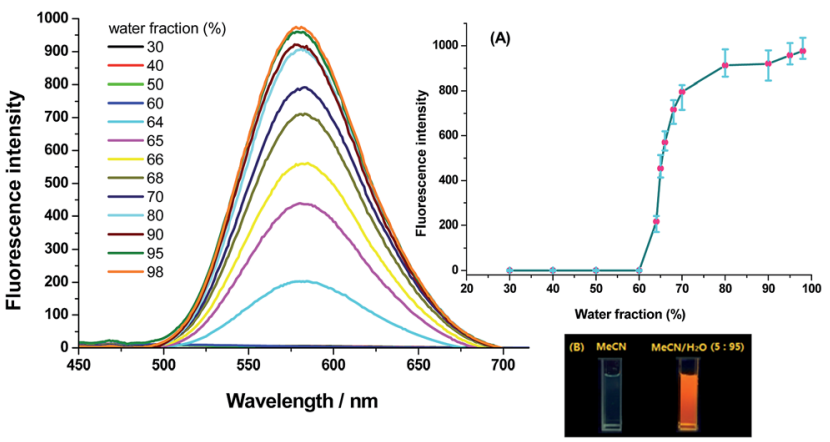

Fig. 3 Emission spectra of $\mathrm{V}_{4}(50 \mu \mathrm{M})$ in $\mathrm{MeCN}$ with varied water fraction [inserted: (A) the fluorescence intensity of $\mathrm{V}_{4}$ in $\mathrm{MeCN}$ with varied water fraction; (B) photos of $\mathrm{V}_{4}$ in $\mathrm{MeCN}$ and $\mathrm{MeCN} / \mathrm{H}_{2} \mathrm{O}(\mathrm{v} / \mathrm{v}$, 5 : 95) mixture].

confirms that the dramatically enhanced fluorescence intensity in aqueous binary mixture with large water content originates from the emission of the aggregated molecules. Additionally, the emission wavelengths of the two compounds in this aqueous mixture (532 $\mathrm{nm}$ for $\mathbf{V}_{2}$ and $580 \mathrm{~nm}$ for $\mathbf{V}_{4}$ ) and in ground samples ( $535 \mathrm{~nm}$ for $\mathbf{V}_{2}$ and $585 \mathrm{~nm}$ for $\mathbf{V}_{4}$ ) are very close to each other, which indicates that out-of-order aggregates are generated in $\mathrm{MeCN} / \mathrm{H}_{2} \mathrm{O}$ mixture, because the ground samples are verified to be in amorphous phase by powder X-ray diffraction (PXRD).

\section{Polymorphism-induced tunable emission}

Unlike in solution, the solid-state fluorescence intimately depends on molecular packing, conformational flexibility and intermolecular interactions, while any modification of molecular arrangement and conformation would inevitably affect the HOMO-LUMO energy levels of a fluorophore and consequently alter its optical property. Hence, polymorphism whose several crystals have dramatically different molecular array and conformation from each other is an important approach to multicolour and tunable emission. ${ }^{16}$ The variations of crystallization conditions like solvents, temperature, concentrations, $\mathrm{pH}$ values and so on strongly influence the ultimate arrangement of molecules and make polymorphism possible.

Through the recrystallization of the compound $\mathbf{V}_{3}$ purified by column chromatography in ethanol under different conditions, two polymorphs with different fluorescence colours are

Table 1 Emission properties of $\mathrm{V}_{1}-\mathrm{V}_{4}$ in different solvents

\begin{tabular}{|c|c|c|c|c|c|c|}
\hline \multirow[b]{2}{*}{ Compd. } & \multicolumn{5}{|l|}{$\lambda_{\mathrm{em}}(\mathrm{nm})$} & \multirow{2}{*}{$\frac{\Phi^{a}}{\text { Acetone }}$} \\
\hline & $n$-Hexane & Benzene & $\mathrm{CH}_{2} \mathrm{Cl}_{2}$ & Acetone & $\mathrm{MeCN}$ & \\
\hline $\mathbf{V}_{2}$ & 459 & 513 & 551 & 578 & 586 & $0.113( \pm 0.008)$ \\
\hline $\mathbf{V}_{3}$ & 460 & 499 & 531 & 548 & 555 & $0.269( \pm 0.017)$ \\
\hline $\mathbf{V}_{4}$ & 490 & 535 & 587 & 605 & 536 & $0.025( \pm 0.004)$ \\
\hline
\end{tabular}

${ }^{a}$ Using quinine sulfate in $0.1 \mathrm{M}$ sulfuric acid solution $(\Phi=0.55)$ as the reference. 
respectively obtained. The green one $\left(\mathbf{V}_{\mathbf{3}}(\mathbf{G})_{\mathbf{C}}\right)$ is generated by dissolving $\mathbf{V}_{3}$ at $50{ }^{\circ} \mathrm{C}$ and cooling it rapidly to $0{ }^{\circ} \mathrm{C}$, while the yellow one $\left(\mathbf{V}_{\mathbf{3}}(\mathbf{Y})_{\mathbf{C}}\right)$ is formed by dissolving $\mathbf{V}_{\mathbf{3}}$ at $80{ }^{\circ} \mathrm{C}$ and slowly cooling it to room temperature. Under an electron microscope, it is clearly shown that $\mathbf{V}_{\mathbf{3}}(\mathbf{Y})_{\mathbf{C}}$ is needle crystals but $\mathbf{V}_{\mathbf{3}}(\mathbf{G})_{\mathbf{C}}$ is flake ones (Fig. 5A and B). Actually, their shapes can be readily distinguished even by the naked eyes. PXRD patterns indicate that they belong to the entirely different crystalline phases whose sharp and intense peaks correspond to distinctive $2 \theta$ angles (Fig. 4A), while the emission wavelengths are respectively centred at $519 \mathrm{~nm}$ for $\mathbf{V}_{\mathbf{3}}(\mathbf{G})_{\mathbf{C}}$ and $535 \mathrm{~nm}$ for $\mathbf{V}_{\mathbf{3}}(\mathbf{Y})_{\mathbf{C}}$
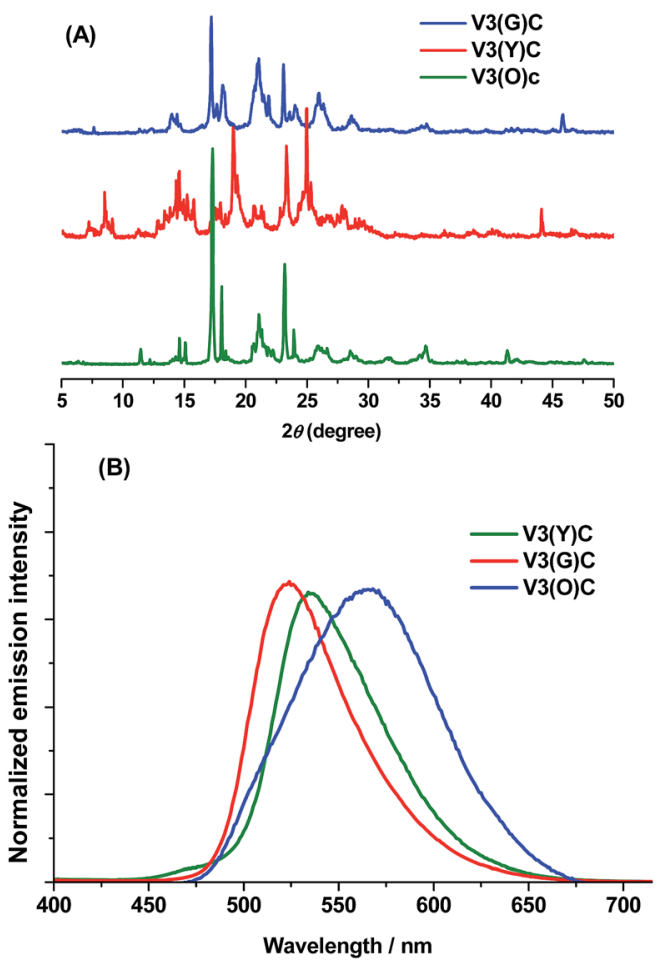

Fig. 4 PXRD patterns (A) and solid-state emission spectra (B) of three crystalline forms of $\mathrm{V}_{3}$.

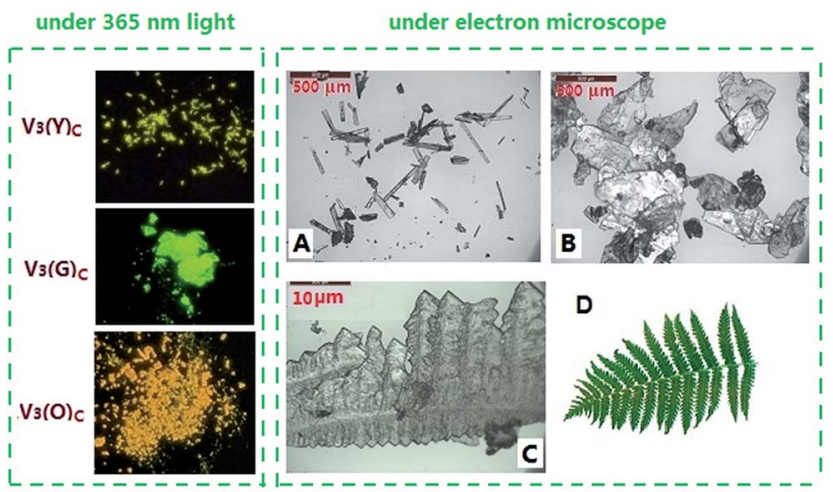

Fig. 5 Photos of three polymorphic forms of $V_{3}$ in crystalline state taken under $365 \mathrm{~nm}$ UV light (left) and electron microscope (right) (A: $\mathrm{V}_{3}(\mathrm{Y}) ; \mathrm{B}: \mathrm{V}_{3}(\mathrm{G}) ; \mathrm{C}: \mathrm{V}_{3}(\mathrm{O})$; $\mathrm{D}$ : a leaf with the similar fractal structure as a $\mathrm{V}_{3}(\mathrm{O})$ particle).
(Fig. 4B). When the pristine $\mathbf{V}_{\mathbf{3}}(\mathbf{Y})_{\mathbf{C}}$ sample is fumed in acetone vapour for $30 \mathrm{~min}$, its fluorescence colour turns from yellow to green. Such change can also be fulfilled by fuming the ground sample $\left(\mathbf{V}_{\mathbf{3}}(\mathbf{Y})_{\mathbf{F C}}\right)$ in acetone vapour for $3 \mathrm{~min}$. Obviously, particles with smaller size are much easier to carry out this conversion. Both PXRD and emission spectra indicate that identical patterns and wavelengths to those of pristine $\mathbf{V}_{\mathbf{3}}(\mathbf{G})_{\mathbf{C}}$ sample occur on the fumed $\mathbf{V}_{\mathbf{3}}(\mathbf{Y})_{\mathbf{F C}}$ or $\mathbf{V}_{\mathbf{3}}(\mathbf{Y})_{\mathbf{C}}$ samples (Fig. S7 and 8, ESI $\dagger$ ), which confirm that a crystalline transformation can be readily achieved from $\mathbf{V}_{\mathbf{3}}(\mathbf{Y})_{\mathbf{C}}$ to $\mathbf{V}_{\mathbf{3}}(\mathbf{G})_{\mathbf{C}}$. However, $\mathbf{V}_{\mathbf{3}}(\mathbf{G})_{\mathbf{C}}$ can be converted into $\mathbf{V}_{\mathbf{3}}(\mathbf{Y})_{\mathbf{C}}$ by neither vapour fuming nor annealing treatment. Moreover, when the melted $\mathbf{V}_{\mathbf{3}}(\mathbf{G})_{\mathbf{C}}$ is either very slowly cooled to room temperature or rapidly quenched in liquid nitrogen, each resulted sample is quite fragile and ready to be crushed into powder whose solid-state emission wavelength is the same as that of the ground $\mathbf{V}_{\mathbf{3}}(\mathbf{G})_{\mathbf{a m}}$ sample (Fig. S9, ESI $\dagger$ ). This result indicates that recrystallization from melted state, which is a common method to realize the transformation between polymorphs, is ineffective for the change from $\mathbf{V}_{\mathbf{3}}(\mathbf{G})_{\mathbf{C}}$ to $\mathbf{V}_{\mathbf{3}}(\mathbf{Y})_{\mathbf{C}}$.

According to Ostwald's step rule, ${ }^{17}$ it is presumed that $\mathbf{V}_{\mathbf{3}}(\mathbf{Y})_{\mathbf{C}}$ should be in thermodynamically more stable state than $\mathbf{V}_{\mathbf{3}}(\mathbf{G})_{\mathbf{C}}$. Based on this assumption, it would be expected that the transformation from $\mathbf{V}_{\mathbf{3}}(\mathbf{G})_{\mathbf{C}}$ to $\mathbf{V}_{\mathbf{3}}(\mathbf{Y})_{\mathbf{C}}$ could smoothly occur by vapour fuming or annealing treatment. However, the conversion between $\mathbf{V}_{\mathbf{3}}(\mathbf{Y})_{\mathbf{C}}$ and $\mathbf{V}_{\mathbf{3}}(\mathbf{G})_{\mathbf{C}}$ does not actually take place in this way. As the thermal gravimetric analysis (TGA) (Fig. S10, ESI $\dagger$ ) indicates that there is only a slight weight loss of $\mathbf{V}_{\mathbf{3}}(\mathbf{G})_{\mathbf{C}}$ until its melting point, it may be safely deduce that $\mathbf{V}_{\mathbf{3}}(\mathbf{G})_{\mathbf{C}}$ is not a pseudopolymorph and its different solid-state emission from that of $\mathbf{V}_{\mathbf{3}}(\mathbf{Y})_{\mathbf{C}}$ should not be caused by the participation of solvent molecules.

Subsequently, thermal properties of $\mathbf{V}_{\mathbf{3}}(\mathbf{G})_{\mathbf{C}}$ and $\mathbf{V}_{\mathbf{3}}(\mathbf{Y})_{\mathbf{C}}$ are investigated by differential scanning calorimetry (DSC) (Fig. S11 $\dagger$ ). It is illustrates that a branched endothermic peak is presented on the curve of $\mathbf{V}_{\mathbf{3}}(\mathbf{G})_{\mathbf{C}}$ whose first tip is centred at $104.5^{\circ} \mathrm{C}$. Below this temperature, none of other peak occurs. Hence, this endothermic peak is assigned as the melting peak of $\mathbf{V}_{\mathbf{3}}(\mathbf{G})_{\mathbf{C}}$. The branches on the peak should be caused by the crystal defects of the sample, as intact crystals of $\mathbf{V}_{\mathbf{3}}(\mathbf{G})_{\mathbf{C}}$ without defects cannot be available for us. On the curve of $\mathbf{V}_{3}(\mathbf{Y})_{C}$, before the melting peak located at the same temperature as that of $\mathbf{V}_{\mathbf{3}}(\mathbf{G})_{\mathbf{C}}$, an additional endothermic peak at $100.1{ }^{\circ} \mathrm{C}$ can be readily observed. This result demonstrates that a phase transformation involving the molecular reorganization from $\mathbf{V}_{\mathbf{3}}(\mathbf{Y})_{\mathbf{C}}$ to $\mathbf{V}_{\mathbf{3}}(\mathbf{G})_{\mathbf{C}}$ really takes place just as what have been verified by PXRD and solid-state emission spectra. Since the $\mathbf{V}_{\mathbf{3}}(\mathbf{Y})_{\mathbf{C}}$ sample for DSC measurement contains particles suitable for single crystal X-ray diffraction, the melting peak on its DSC curve is completely convergent without any branch. The DSC curves reveal that $\mathbf{V}_{\mathbf{3}}(\mathbf{Y})_{\mathbf{C}}$ and $\mathbf{V}_{\mathbf{3}}(\mathbf{G})_{\mathbf{C}}$ are enantiotropic polymorphs, but the one-way phase change from the presumably low energy $\mathbf{V}_{\mathbf{3}}(\mathbf{Y})_{\mathbf{C}}$ to the high energy $\mathbf{V}_{\mathbf{3}}(\mathbf{G})_{\mathbf{C}}$ is rarely unusual and seems to be related to their molecular packing modes (vide infra).

Interestingly, the fluorescence colour further turns into orange in $565 \mathrm{~nm}$ (Fig. 4B) when the ground $\mathbf{V}_{\mathbf{3}}(\mathbf{Y})_{\mathbf{F C}}$ sample is fumed for $30 \mathrm{~min}$. Similar case also occurs on the ground 
$\mathbf{V}_{\mathbf{3}}(\mathbf{G})_{\mathbf{a m}}$ sample. PXRD pattern demonstrates that the orange sample $\left(\mathbf{V}_{\mathbf{3}}(\mathbf{O})_{\mathbf{C}}\right)$ is also in crystalline state, and its peaks are very similar to those of the pristine $\mathbf{V}_{\mathbf{3}}(\mathbf{G})_{\mathbf{C}}$ sample (Fig. $\left.4 \mathrm{~A}\right)$. Obviously, the difference of emission between $\mathbf{V}_{\mathbf{3}}(\mathbf{O})_{\mathbf{C}}$ and $\mathbf{V}_{\mathbf{3}}(\mathbf{G})_{\mathbf{C}}$ should not be attributed to their different conformations in crystalline phase. However, $\mathbf{V}_{\mathbf{3}}(\mathbf{O})_{\mathbf{C}}$ cannot be gained by annealing of $\mathbf{V}_{\mathbf{3}}(\mathbf{Y})_{\mathbf{F C}}$ or $\mathbf{V}_{\mathbf{3}}(\mathbf{G})_{\mathbf{a m}}$, even if the treatment time is largely prolonged.

In addition to polymorphism involving molecules with different conformations, the other important approach to tunable solid-state emission is micro-/nano-fabrication, by which crystals with the same molecular conformations are assembled into micro-/nano-scaled particles in specific shapes. ${ }^{18}$ Hence, we are encouraged to investigate the shapes of $\mathbf{V}_{\mathbf{3}}(\mathbf{O})_{\mathbf{C}}$ particles, as $\mathbf{V}_{\mathbf{3}}(\mathbf{O})_{\mathbf{C}}$ and $\mathbf{V}_{\mathbf{3}}(\mathbf{G})_{\mathbf{C}}$ have the same PXRD patterns but display the different fluorescence colours. The photo of a $\mathbf{V}_{3}(\mathbf{O})_{\mathbf{C}}$ crystalline particle taken under an electron microscope (Fig. 5C) clearly shows that branches with sharp dentate tips are regularly arranged into a kind of fractal structure, where the growth lines are readily observable, just like the veins on a leaf with the similar fractal geometry (Fig. 5D). Very possibly, it is such particular shape that makes $\mathbf{V}_{\mathbf{3}}(\mathbf{O})_{\mathbf{C}}$ emit orange fluorescence.

A reasonable hypothesis for the micro-fabrication of $\mathbf{V}_{\mathbf{3}}(\mathbf{O})_{\mathbf{C}}$ involves a dynamic dissolution-recrystallization process. Under short-time vapour fuming, solvent molecules can just diffuse inward into $\mathbf{V}_{\mathbf{3}}(\mathbf{Y})_{\mathbf{F C}}$ or $\mathbf{V}_{\mathbf{3}}(\mathbf{G})_{\mathbf{a m}}$ particles but not dissolve them, offering $\mathbf{V}_{\mathbf{3}}$ molecules some extent of mobility to be reorganized into $\mathbf{V}_{\mathbf{3}}(\mathbf{G})_{\mathbf{C}}$. In prolonged exposure to vapour, the formed tiny $\mathbf{V}_{\mathbf{3}}(\mathbf{G})_{\mathbf{C}}$ crystals are gradually dissolved in the vapour. When solvent molecules leave off, $\mathbf{V}_{\mathbf{3}}$ molecules are preferably recrystallized and self-assembled into $\mathbf{V}_{\mathbf{3}}(\mathbf{O})_{\mathbf{C}}$ with unique fractal structure. As annealing treatment below melting point cannot provide particles with the sufficient mobility to make macroscopically morphological change, $\mathbf{V}_{\mathbf{3}}(\mathbf{O})_{\mathbf{C}}$ crystals are unable to be obtained by annealing of $\mathbf{V}_{\mathbf{3}}(\mathbf{Y})_{\mathbf{F C}}$ or $\mathbf{V}_{\mathbf{3}}(\mathbf{G})_{\mathbf{a m}}$ powder.

\section{Solid-state emission under force stimuli}

Due to the lack of strong intermolecular interactions like $\pi-\pi$ overlaps, $\mathbf{V}_{\mathbf{1}}-\mathbf{V}_{\mathbf{4}}$ exhibit intensely solid-state emission whose quantum yields determined by using an integrating sphere are $0.434( \pm 0.021)\left(\mathbf{V}_{\mathbf{1}}\right), 0.565( \pm 0.025)\left(\mathbf{V}_{\mathbf{2}}\right), 0.621( \pm 0.027)\left(\mathbf{V}_{\mathbf{3}}(\mathbf{G})_{\mathbf{C}}\right)$, $0.513( \pm 0.035)\left(\mathbf{V}_{3}(\mathbf{Y})_{\mathbf{C}}\right), 0.335( \pm 0.018)\left(\mathbf{V}_{\mathbf{3}}(\mathbf{O})_{\mathbf{C}}\right)$ and 0.317 $( \pm 0.020)\left(\mathbf{V}_{4}\right)$, respectively. Similar to that in solution state, the pristine $\mathbf{V}_{\mathbf{1}}$ and $\mathbf{V}_{\mathbf{4}}$ samples in solid state correspondingly has the shortest and longest emission wavelength. Different from that in solution state, however, both the as-prepared $\mathbf{V}_{\mathbf{3}}(\mathbf{G})_{\mathbf{C}}$ and $\mathbf{V}_{\mathbf{3}}(\mathbf{Y})_{\mathbf{C}}$ samples display longer emission wavelengths than $\mathbf{V}_{\mathbf{2}}$. Apparently, intermolecular interactions in solid state should account for such difference. For pristine $\mathbf{V}_{\mathbf{1}}, \mathbf{V}_{\mathbf{2}}, \mathbf{V}_{\mathbf{4}}$ and $\mathbf{V}_{\mathbf{3}}(\mathbf{G})_{\mathbf{C}}$ samples, each of them is MFC-active with vividly contrasted color change before and after force stimuli. Under exposure to force stimuli, a largely wide and nearly successive range of solidstate emission wavelengths can be achieved on these V-shaped fluorophores by subtle structure modification (Fig. 6). However, it seems that their emission shifts (30-40 nm) under force

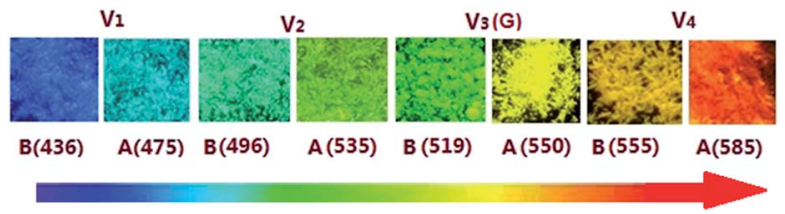

Fig. 6 Photos of $V_{1}-V_{4}$ before and after grinding under $365 \mathrm{~nm}$ UV light (B: before grinding; $A$ : after grinding; unit: $n m$ ).

stimuli are related to neither the number nor the position of vinyl. The detailed experimental and theoretic research in our previous work has verified that not only the extent of ICT effect at single molecular level but also intermolecular interactions at supramolecular level have profound impact on MFC activity of a V-shaped fluorophore. Therefore, it seems that some unknown compromises between ICT extent and intermolecular interactions result in the apparently structure-irrelevant emission shift in this family.

In order to have a deep insight of the MFC activity in this family, PXRD measurements are carried out on the samples of $\mathbf{V}_{\mathbf{1}}-\mathbf{V}_{\mathbf{4}}$. The corresponding patterns of $\mathbf{V}_{\mathbf{1}}, \mathbf{V}_{\mathbf{2}}$ and $\mathbf{V}_{\mathbf{4}}$ (Fig. S12-14, ESI $\dagger$ ) clearly indicate that transformations from crystalline to amorphous phase take place on them by grinding, and such morphology-dependent conversion can be reversibly recovered with robust fatigue resistance via fuming or annealing the ground samples, providing the restored samples with the emission wavelengths nearly identical to those of the asprepared ones (Fig. S15-17, ESI $\dagger$ ). The similar case occurs on $\mathbf{V}_{\mathbf{3}}(\mathbf{G})_{\mathbf{C}}$ (Fig S18 and 19, ESI $\dagger$ ) but not on $\mathbf{V}_{\mathbf{3}}(\mathbf{Y})_{\mathbf{C}}$, because the ground $\mathbf{V}_{\mathbf{3}}(\mathbf{Y})_{\mathbf{F C}}$ presents the same emission spectra as the pristine $\mathbf{V}_{\mathbf{3}}(\mathbf{Y})_{\mathbf{C}}$ (Fig. S8, ESI $\dagger$ ). The PXRD pattern (Fig. S7, ESI $\dagger$ ) demonstrates that a crystalline phase is still maintained by $\mathbf{V}_{\mathbf{3}}(\mathbf{Y})_{\mathbf{F C}}$ even if it is vigorously and thoroughly ground. Since $\mathbf{V}_{\mathbf{3}}(\mathbf{G})_{\mathbf{C}}$ and $\mathbf{V}_{\mathbf{3}}(\mathbf{Y})_{\mathbf{C}}$ polymorphs have the same chemical structure, the MFC inertia of $\mathbf{V}_{\mathbf{3}}(\mathbf{Y})_{\mathbf{C}}$ should be attributed to its particular packing mode, which is confirmed by the X-ray crystallographic analysis (vide infra). Moreover, the emission wavelength of the ground $\mathbf{V}_{\mathbf{3}}(\mathbf{O})_{\text {am }}$ sample makes a unique blue shift (Fig. S20, ESI $\dagger$ ) due to the destruction of the fractal structure, which is equivalent to that of $\mathbf{V}_{\mathbf{3}}(\mathbf{G})_{\mathbf{a m}}$. By fuming $\mathbf{V}_{\mathbf{3}}(\mathbf{O})_{\mathbf{a m}}$ for $1 \mathrm{~min}$ or annealing it for $3 \mathrm{~min}$, its emission wavelength is further hypsochromically shifted to be identical to that of $\mathbf{V}_{\mathbf{3}}(\mathbf{G})_{\mathbf{C}}$. PXRD patterns (Fig. S21, ESI $\dagger$ ) verify that the ordered structures identical to $\mathbf{V}_{\mathbf{3}}(\mathbf{G})_{\mathbf{C}}$ are completely recovered on the fumed or annealed $\mathbf{V}_{\mathbf{3}}(\mathbf{O})_{\mathbf{a m}}$ sample. For $\mathbf{V}_{\mathbf{3}}(\mathbf{O})_{\mathbf{a m}}$, it can be smoothly converted into $\mathbf{V}_{\mathbf{3}}(\mathbf{O})_{\mathbf{C}}$ by prolonged exposure to solvent vapour, just as the cases happen on $\mathbf{V}_{\mathbf{3}}(\mathbf{Y})_{\mathbf{F C}}$ or $\mathbf{V}_{\mathbf{3}}(\mathbf{G})_{\mathbf{a m}}$. Herein, a schematic diagram which describes the transformations among various forms of $\mathbf{V}_{\mathbf{3}}$ under different conditions is summarily presented in Fig. 7 .

The films made by spreading out the tiny crystalline particles of these MFC-active compounds on filtrate paper can be used as the erasable mediums for information storage (Fig. 8). Initially, two letters "Z" and "J" in vivid contrast from the background are written on the films by using a thin glass rod. In order to produce the readily observable letters on the films, the places on 


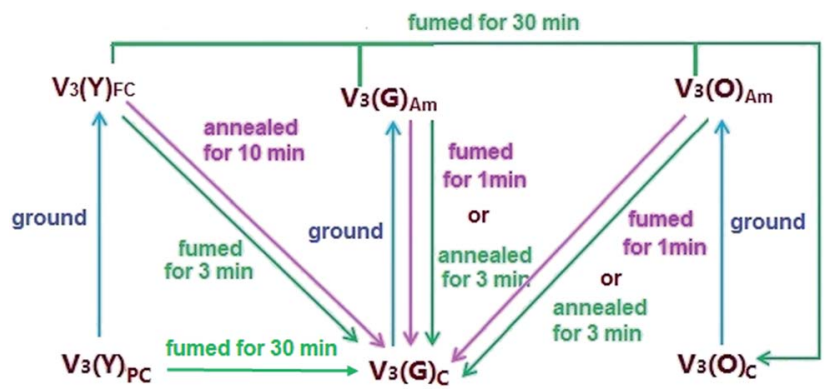

Fig. 7 Schematic diagram of transformations among different forms of $\mathrm{V}_{3}$ (C: in crystalline phase; FC: in crystalline phase with fine particles: PC: in pristine crystalline phase; Am: in amorphous phase).

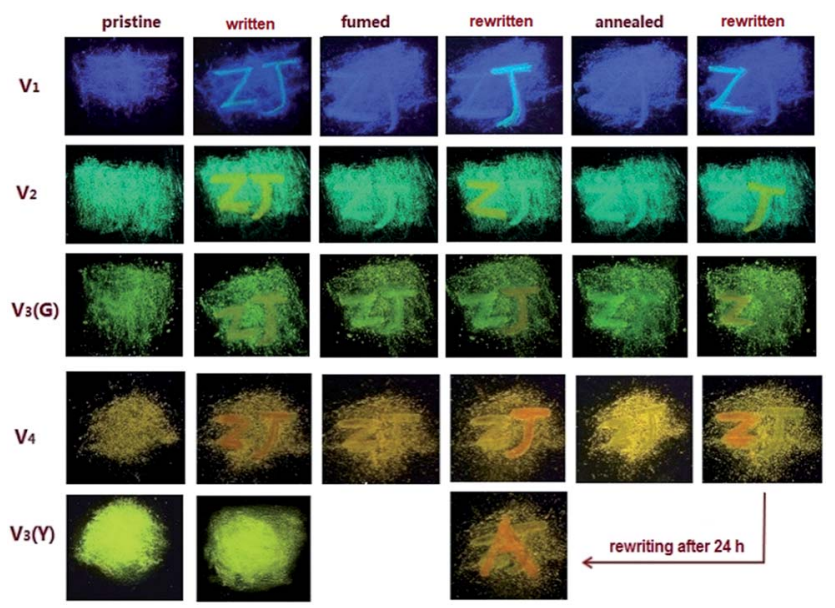

Fig. 8 Photos of letters written on compound films taken under $365 \mathrm{~nm}$ UV light.

which the letters are written have to be repeatedly smeared by the rod, making the writing traces stay there. After the films are fumed in acetone vapor, the colors of the letters are fully restored into those of the backgrounds, even if the writing traces are still remained. Subsequently, the letter "Z" or "J" can be rewritten on the fumed films, whose colors are the same as that of the initially written ones. After annealing the films at $80^{\circ} \mathrm{C}$, the colors of the rewritten letters are completely faded again into those of the backgrounds, and the third-time writing of letter "Z" or "J" can be easily carried out on the annealed films. Actually, the above writing-erasing process is able to be repeated for at least 20 times without obvious fatigue. However, any color change does not occur on the $\mathbf{V}_{3}(\mathbf{Y})_{C}$ film under the similar treatment.

For all the MFC-responsive members, the time consumed by the ground samples to be fully recovered into crystalline phase is much shorter via annealing than via fuming, which means that solvent vapour is more efficient than heat to rebuild the ordered structure. Additionally, it takes each compound different time to be restored from amorphous phase. The time required by fuming is $30 \mathrm{~min}$ for $\mathbf{V}_{\mathbf{1}}, \mathbf{1} \mathrm{min}$ for $\mathbf{V}_{\mathbf{2}}$ and $\mathbf{V}_{\mathbf{3}}(\mathbf{G})_{\mathrm{C}}$, and just $10 \mathrm{~s}$ for $\mathbf{V}_{\mathbf{4}}$, while the time needed by annealing is $1 \mathrm{~h}$ for $\mathbf{V}_{\mathbf{1}}, 2-3 \mathrm{~min}$ for $\mathbf{V}_{\mathbf{2}}$ and $\mathbf{V}_{\mathbf{3}}(\mathbf{G})_{\mathbf{C}}$, and only $30 \mathrm{~s}$ for $\mathbf{V}_{\mathbf{4}}$. Actually, the recovery of $\mathbf{V}_{\mathbf{4}}$ is so ready that an isothermal phase transformation can even spontaneously take place at room temperature. The solid-state emission spectra (Fig. S22, ESI $\dagger$ ) show that the emission wavelength of the ground $\mathbf{V}_{\mathbf{4}}$ sample is progressively blue-shifted until it is the same as that of the pristine sample after $24 \mathrm{~h}$. Such automatic recovery can be directly observed on the $\mathbf{V}_{\mathbf{4}}$ film with the already written letter "Z", where another letter "A" can be smoothly rewritten on it after it is stood at room temperature for $24 \mathrm{~h}$ (Fig. 8). These results indicate that the existence of vinyl facilitates the recovery of corresponding samples from random to ordered structure.

\section{X-ray crystallographic analysis}

In addition to PXRD measurements, X-ray crystallographic analysis is another important way to explore the nature of the MFC activity in this family. Herein, high quality crystals of $\mathbf{V}_{\mathbf{1}}, \mathbf{V}_{\mathbf{2}}$ and $\mathbf{V}_{3}(\mathbf{Y})_{\mathbf{C}}$ are gained by recrystallization of corresponding compound in EtOH/MeCN mixture. However, $\mathbf{V}_{\mathbf{3}}(\mathbf{G})_{\mathbf{C}}$ crystal with sufficient thickness and no defect is unable to be available and $\mathbf{V}_{\mathbf{4}}$ one is full of cracks even if it has large enough 3D size. Hence, the satisfactory crystallographic data are obtained for neither $\mathbf{V}_{\mathbf{3}}(\mathbf{G})_{\mathbf{C}}$ nor $\mathbf{V}_{\mathbf{4}}$ by our best effort.

Fig. 9 demonstrates that several weak second-order forces like $\mathrm{C}-\mathrm{H} \cdots \pi$ interactions and $\mathrm{C}-\mathrm{H} \cdots \mathrm{N}$ hydrogen bonds rather than $\pi-\pi$ overlaps should be responsible for the efficient immobilization of the conformations in $\mathbf{V}_{\mathbf{1}}, \mathbf{V}_{\mathbf{2}}$ and $\mathbf{V}_{\mathbf{3}}(\mathbf{Y})_{\mathbf{C}}$ crystals. X-ray diffraction reveals that $\mathrm{H}$-bonds participated by $\mathrm{N}$ atoms on dimethylamino-phenyls emerge in each of these crystals, and it is suggested that the collapse of lattices and the destruction of $\mathrm{H}$-bonds due to the protonation at these $\mathrm{N}$ atoms should account for the liquidation of them into films with the quenched fluorescence when they are exposed to trifluoroacetic acid (TFA) vapour. As $\mathbf{V}_{\mathbf{4}}$ has the similar structure to those of $\mathbf{V}_{\mathbf{1}^{-}}$ $\mathbf{V}_{\mathbf{3}}$, it is safely to deduce that the liquidation of $\mathbf{V}_{\mathbf{4}}$ in presence of TFA vapour may well be attributed to the same reason as that for liquidation of $\mathbf{V}_{\mathbf{1}}-\mathbf{V}_{\mathbf{3}}$, even if the crystallographic data of $\mathbf{V}_{\mathbf{4}}$ is lack under the current condition.

For $\mathbf{V}_{\mathbf{1}}, \mathbf{V}_{\mathbf{2}}$ and $\mathbf{V}_{\mathbf{3}}(\mathbf{Y})_{\mathbf{C}}$ crystals, each of them belongs to the monoclinic crystal system and the $P 2_{1} / c$ space group. In $\mathbf{V}_{\mathbf{1}}$ crystal, the adjacent (benzo[d]thiazol-2-yl)phenyl moieties are oppositely approached along the $r_{2}$ direction via double $\mathrm{H}$ bonds $(2.713 \AA)$, producing "open" substructures with outward dimethyl-aminophenyls. Meanwhile, the adjacent molecules along the $r_{1}$ direction interact with each other via $\mathrm{C}-\mathrm{H} \cdots \mathrm{N}$ hydrogen bond and the electron-deficient moiety are quite planar. Consequently, it is reasonable to deduce that the damages of the intermolecular interactions due to the planarization of conformation induced by the rotation of dimethylaminophenyl under force stimuli should account for the redshifted emission of the amorphous samples. For a $\mathbf{V}_{\mathbf{3}}(\mathbf{Y})_{\mathbf{C}}$ crystal, however, it is packed by special molecular pairs (note: they are not dimers formed via intermolecular interactions between two crystallographically equivalent adjacent molecules like the cases in $\mathbf{V}_{\mathbf{1}}$ and $\mathbf{V}_{\mathbf{2}}$ crystals). In each pair, the double crystallo-graphically non-equivalent molecules interact with each other via two forms of $\mathrm{C}-\mathrm{H} \cdots \pi$ forces $(2.702$ and $3.224 \AA)$, 
(A)
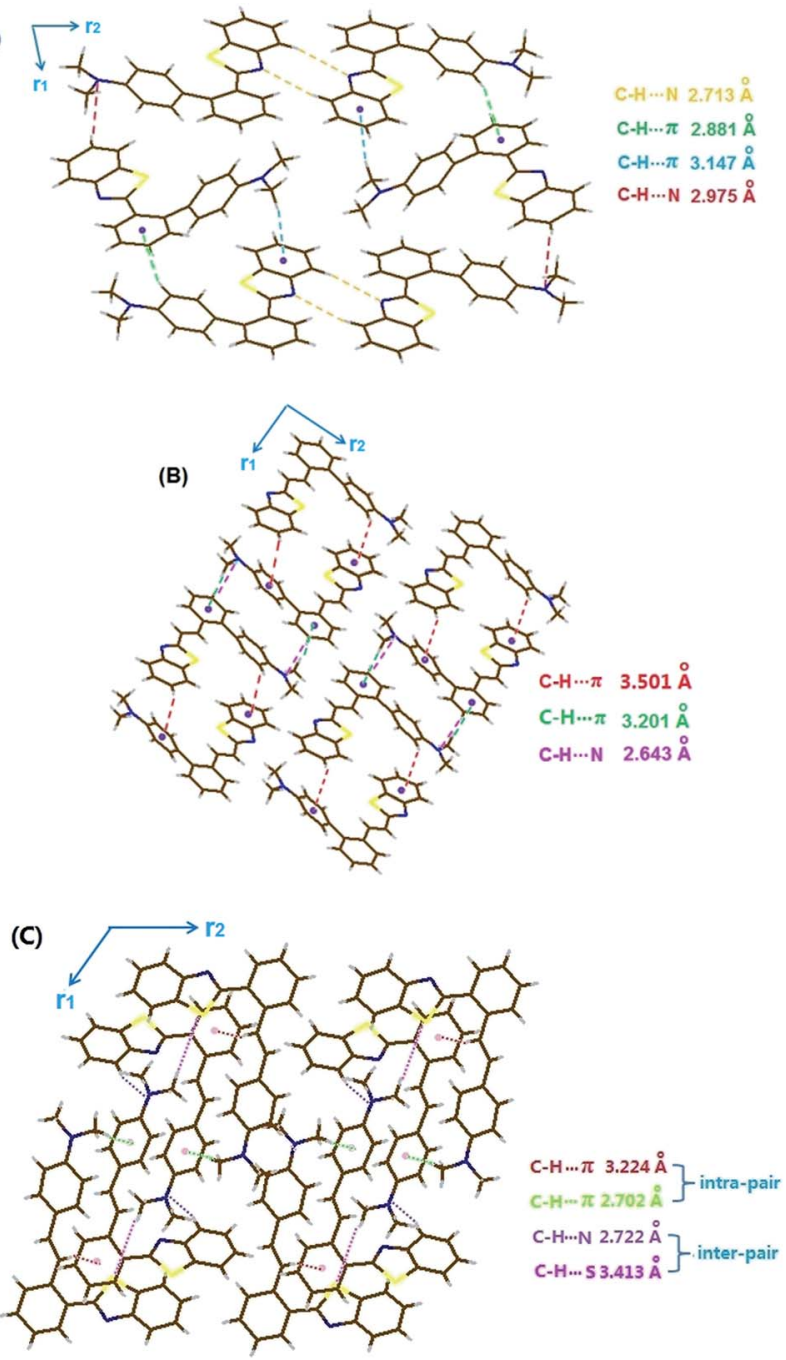

Fig. 9 Molecular stacking of $\mathrm{V}_{1}(\mathrm{~A}), \mathrm{V}_{2}(\mathrm{~B})$ and $\mathrm{V}_{3}(\mathrm{Y})(\mathrm{C})$.

which make the global conformation of the both molecules still largely distorted and the opposite orientation of the two 2-benzo $[d]$ thiazolyls in crystalline phase (Fig. S23C, ESI $\dagger$ ). If a pair is treated as a molecule, the similar packing mode to that of $\mathbf{V}_{\mathbf{2}}$ occurs in $\mathbf{V}_{\mathbf{3}}(\mathbf{Y})_{\mathbf{C}}$ with the opposite arrangement of 2-benzo[d] thiazolyl along the $r_{1}$ direction via two $\mathrm{H}$-bonds $(\mathrm{C}-\mathrm{H} \cdots \mathrm{N}$ in $2.722 \AA$ and $\mathrm{C}-\mathrm{H} \cdots \mathrm{S}$ in $3.413 \AA$ ), producing the "closed" tubeshaped substructures with inward dimethylaminophenyl-4styryls. In lack of the interactions along the $r_{2}$ direction, the pairs are stacked into 1D chains. Fully different from the break of interactions between the adjacent individual molecules in both $\mathbf{V}_{\mathbf{1}}$ and $\mathbf{V}_{\mathbf{2}}$ crystals, it is assumed that only the interactions between the adjacent pairs rather than those inside pairs can be destroyed under force stimuli in $\mathbf{V}_{\mathbf{3}}(\mathbf{Y})_{\mathbf{C}}$ crystal. Hence, the undamaged intermolecular interactions make the conformation change hardly take place inside pairs, which results in the heavy resistance of $\mathbf{V}_{\mathbf{3}}(\mathbf{Y})_{\mathbf{C}}$ sample against force disruption.

Although the crystallographic data of $\mathbf{V}_{\mathbf{3}}(\mathbf{G})_{\mathbf{C}}$ is unavailable for us at present, it is deduced that the molecular packing in this crystal may well resemble that in $\mathbf{V}_{\mathbf{1}}$ and $\mathbf{V}_{\mathbf{2}}$ participated by individual molecules, due to their similar MFC behaviours. The abnormal transformation from presumably lower energy $\mathbf{V}_{\mathbf{3}}(\mathbf{Y})_{\mathbf{C}}$ into higher energy $\mathbf{V}_{\mathbf{3}}(\mathbf{G})_{\mathbf{C}}$ reminds us of the similar case taken place on the two polymorphs of 2-octyl-6-[(4(octyloxy)naphthalen-1-yl)ethynyl]-1 $H$-benzo[de]isoquinoline1,3(2H)-dione. ${ }^{19}$ In this report, it was suggested that some structural link between dynamic motion and stability of a certain packing mode should account for the abnormal polymorph change from lower to higher energy state. Hence, we are enlightened to consider that the packing in $\mathbf{V}_{\mathbf{3}}(\mathbf{Y})_{\mathbf{C}}$ established by the unique molecular pairs seems to be the possible reason for the unusual conversion from a presumably stable to a metastable crystal in our work, as a polymorph crystallized by molecular pairs is very rare in most of polymorphic systems. However, the exact nature for this peculiar transformation from $\mathbf{V}_{\mathbf{3}}(\mathbf{Y})_{\mathbf{C}}$ to $\mathbf{V}_{\mathbf{3}}(\mathbf{G})_{\mathbf{C}}$ is still currently unknown.

\section{Conclusion}

Herein we describe the synthesis and photophysical characteristics of some V-shaped fluorophores, on which varied number of vinyl is introduced at different position of the skeleton. These compounds, which are intensely emissive in solid state and sensitive to force stimuli, can be gained in good yields by using readily available starting materials via a straightforward and reliable synthetic route. The structureproperty relationship reveals that many physical features of this family are closely related to the number and position of vinyl: (1) solid-state emission wavelengths, solvota-chromic effect, solution-state quantum yields and AIE activity heavily depend on both the number and the site of vinyl; (2) solutionstate emission wavelengths and time consumed for samples to be restored from amorphous to crystalline phase only rely on the number rather than the location of vinyl; (3) emission wavelength shifts before and after force stimuli seem to be dependent on neither the number nor the position of vinyl. By the subtle structure modification, a largely wide and approximately successive range of solid-state emission wavelengths can be achieved on these V-shaped fluorophores in exposure to force stimuli.

Except for MFC effect induced by morphologic change, $\mathbf{V}_{\mathbf{3}}$ turns up tunable fluorescence colors by means of polymorphism and shape-dependent micro-fabrication, by which the unique fractal structure of particles is probably produced by the self-assembling of $\mathbf{V}_{\mathbf{3}}$ molecules through a dissolutionrecrystallization process. It is suggested that the unbroken intermolecular interactions inside the particular molecular pairs may well account for the resistance of $\mathbf{V}_{3}(\mathbf{Y})_{\mathbf{C}}$ crystal against force stimuli. Although it is presumed that the abnormal phase transformation from apparently stable $\mathbf{V}_{\mathbf{3}}(\mathbf{Y})_{\mathbf{C}}$ to metastable $\mathbf{V}_{\mathbf{3}}(\mathbf{G})_{\mathbf{C}}$ may also be ascribed to these molecular pairs, the real cause for such transformation is still unclear. Future studies will continue to probe this question and the development of $\mathbf{V}_{\mathbf{3}}(\mathbf{O})_{\mathbf{C}}$ with attractive fractal structure as promising material in optoelectronic field. 


\section{Conflicts of interest}

There are no conflicts of interest to declare in this work.

\section{Acknowledgements}

We are grateful for the financial support from Zhejiang Provincial Top Key Academic Discipline of Chemical Engineering and Technology.

\section{References}

1 (a) A. Beneduci, S. Cospito, M. La Deda, L. Veltri and G. Chidichimo, Nat. Commun., 2014, 5, 3105; (b) W. R. Browne, M. M. Pollard, B. De Lange, A. Meetsma and B. L. Feringa, J. Am. Chem. Soc., 2006, 128, 12412-12413; (c) A. Seeboth, D. Lotzsch and R. Ruhmann, J. Mater. Chem. C, 2013, 1, 2811-2816; (d) X. Luo, W. Zhao, J. Shi, C. Li, Z. Liu, Z. Bo, Y. Q. Dong and B. Z. Tang, J. Phys. Chem. C, 2012, 116, 21967-21972; (e) E. Takahashi, H. Takaya and T. Naota, Chem.-Eur. J., 2010, 16, 4793-4802.

2 (a) Y. Q. Dong, J. W. Y. Lam and B. Z. Tan, J. Phys. Chem. Lett., 2015, 6, 3429-3436; (b) Z. Chi, X. Zhang, B. Xu, X. Zhou, C. Ma, Y. Zhang, S. Liu and J. Xu, Chem. Soc. Rev., 2012, 41, 3878-3896; (c) S. Xue, X. Qiu, Q. Sun and W. Yang, J. Mater. Chem. C, 2016, 4, 1568-1578; (d) C. Wang and Z. Li, Mater. Chem. Front., 2017, 1, 2174-2194.

3 (a) A. Pucci, F. D. Cuia, F. Signori and G. Ruggeri, J. Mater. Chem., 2007, 17, 783-790; (b) Y. Dong, J. W. Y. Lam, A. Qin, J. Liu, Z. Li, B. Z. Tang, J. Sun and H. S. Kwok, Appl. Phys. Lett., 2007, 91, 011111; (c) Z. Ning, Z. Chen, Q. Zhang, Y. Yan, S. Qian, Y. Cao and H. Tian, Adv. Funct. Mater., 2007, 17, 3799-3807; (d) B. R. Crenshaw, M. Burnworth, D. Khariwala, A. Hiltner, P. T. Mather, R. Simha and C. Weder, Macromolecules, 2007, 40, 2400-2408; (e) M. Kinami, B. R. Crenshaw and C. Weder, Chem. Mater., 2006, 18, 946-955; (f) S. J. Toal, K. A. Jones, D. Magde and W. C. Trogler, J. Am. Chem. Soc., 2005, 127, 11661-11665; (g) W. Chen, E. W. Rosser, D. Zhang, W. Shi, Y. Li, W.-J. Dong, H. Ma, D. Hu and M. Xian, Org. Lett., 2015, 17, 2776-2779; (h) R. Zhang, C.-J. Zhang, Z. Song, J. Liang, R. T. K. Kwok, B. Z. Tang and B. Liu, J. Mater. Chem. C, 2016, 4, 2834-2842.

4 (a) S. Hirata and T. Watanabe, Adv. Mater., 2006, 18, 27252729; (b) S. J. Lim, B. K. An, S. D. Jung, M. A. Chung and S. Y. Park, Angew. Chem., Int. Ed., 2004, 43, 6346-6350; (c) C. E. Olson, M. J. R. Previte and J. T. Fourkas, Nat. Mater., 2002, 1, 225-228; (d) M. Irie, T. Fukaminato, T. Sasaki, N. Tamai and T. Kawai, Nature, 2002, 420, 759-760.

5 (a) A. Kishimura, T. Yamashita, K. Yamaguchi and T. Aida, Nat. Mater., 2005, 4, 546-549; (b) X. Zhu, R. Liu, Y. Li, H. Huang, Q. Wang, D. Wang, X. Zhu, S. Liu and H. Zhu, Chem. Commun., 2014, 50, 12951-12954; (c) Q. Qi, Y. Liu, X. Fang, Y. Zhang, P. Chen, Y. Wang, B. Yang, B. Xu, W. Tian and S. X.-A. Zhang, RSC Adv., 2013, 3, 7996-8002; (d) P. Kumar, J. Dwivedi and B. K. Gupta, J. Mater. Chem. C, 2014, 2, 10468-10475.
6 (a) W. Z. Yuan, Y. Tan, Y. Gong, P. Lu, J. W. Y. Lam, X. Y. Shen, C. Feng, H. Y. Sung, Y. Lu, I. D. Williams, J. Z. Sun, Y. Zhang and B. Z. Tang, Adv. Mater., 2013, 25, 2837-2843; (b) C. Li, X. Tang, L. Zhang, C. Li, Z. Liu, Z. Bo, Y. Q. Dong, Y.-H. Tian, Y. Dong and B. Z. Tang, Adv. Opt. Mater., 2015, 3, 1184-1190; (c) K. C. Naeem, A. Subhakumari, S. Varughese and V. C. Nair, J. Mater. Chem. C, 2015, 3, 10225-10231.

7 (a) Y. Sagara and T. Kato, Angew. Chem., Int. Ed., 2011, 50, 9128-9132; (b) S.-J. Yoon, J. W. Chung, J. Gierschner, K. S. Kim, M.-G. Choi, D. Kim and S. Y. Park, J. Am. Chem. Soc., 2010, 132, 13675-13683; (c) H. Sun, S. Liu, W. Lin, K. Y. Zhang, W. Lv, X. Huang, F. Huo, H. Yang, G. Jenkins, Q. Zhao and W. Huang, Nat. Commun., 2014, 5, 3601-3609; (d) K. Y. Zhang, S. Liu, Q. Zhao and W. Huang, Coord. Chem. Rev., 2016, 319, 180-195; (e) X. Chen, G. Sun, T. Zhang, S. Liu, Q. Zhao and W. Huang, Adv. Mater., 2016, 28, 7137-7142; $(f)$ Q. Zhao, W. Xu, H. Sun, J. Yang, K. Y. Zhang, S. Liu, Y. Ma and W. Huang, Adv. Opt. Mater., 2016, 4, 1167-1173; (g) W. Lin, Q. Zhao, H. Sun, K. Y. Zhang, H. Yang, Q. Yu, X. Zhou, S. Guo, S. Liu and W. Huang, Adv. Opt. Mater., 2015, 3, 368-375; (h) J. Han, J. Sun, Y. Li, Y. Duan and T. Han, J. Mater. Chem. C, 2016, 4, 9287-9293.

8 (a) X. Zhang, Z. Chi, J. Zhang, H. Li, B. Xu, X. Li, S. Liu, Y. Zhang and J. Xu, J. Phys. Chem. B, 2011, 115, 7606-7611; (b) P. Xue, B. Yao, J. Sun, Q. Xu, P. Chen, Z. Zhang and R. Lu, J. Mater. Chem. C, 2014, 2, 3942-3950; (c) Y. Gong, Y. Tan, J. Liu, P. Lu, C. Feng, W. Z. Yuan, Y. Lu, J. Z. Sun, G. He and Y. Zhang, Chem. Commun., 2013, 49, 4009-4011; (d) R. Misra, T. Jadhav, B. Dhokale and S. M. Mobin, Chem. Commun., 2014, 50, 9076-9078; (e) X. Cheng, H. Zhang, K. Ye, H. Zhang and Y. Wang, J. Mater. Chem. C, 2013, 1, 7507-7512.

9 (a) L. Bu, M. Sun, D. Zhang, W. Liu, Y. Wang, M. Zheng, S. Xue and W. Yang, J. Mater. Chem. C, 2013, 1, 2028-2035; (b) M. Zheng, D. T. Zhang, M. X. Sun, Y. P. Li, T. L. Liu, S. F. Xue and W. J. Yang, J. Mater. Chem. C, 2014, 2, 19131920; (c) H. Luo, S. Chen, Z. Liu, C. Zhang, Z. Cai, X. Chen, G. Zhang, Y. Zhao, S. Decurtins, S.-X. Liu and D. Zhang, Adv. Funct. Mater., 2014, 24, 4250-4258; (d) M. A. Saeed, H. T. M. Le and O. Š. Miljanić, Acc. Chem. Res., 2014, 47, 2074-2083; (e) X. Zhang, Z. Chi, J. Zhang, H. Li, B. Xu, X. Li, S. Liu, Y. Zhang and J. Xu, J. Phys. Chem. B, 2011, 115, 7606-7611; (f) J. N. Zhang, H. Kang, N. Li, S. M. Zhou, H. M. Sun, S. W. Yin, N. Zhao and B. Z. Tang, Chem. Sci., 2017, 8, 577-582.

10 (a) H. Li, X. Zhang, Z. Chi, B. Xu, W. Zhou, S. Liu, Y. Zhang and J. Xu, Org. Lett., 2011, 13, 556-559; (b) J. Sun, Y. Dai, M. Ouyang, Y. Zhang, L. Zhan and C. Zhang, J. Mater. Chem. C, 2015, 3, 3356-3363; (c) J. Sun, X. Lv, P. Wang, Y. Zhang, Y. Dai, Q. Wu, M. Ouyang and C. Zhang, J. Mater. Chem. C, 2014, 2, 5365-5371.

11 X.-L. Lu and M. Xia, J. Mater. Chem. C, 2016, 4, 9350-9358. 12 H.-Y. Fu, N. Xu, Y.-M. Pan, X.-L. Lu and M. Xia, Phys. Chem. Chem. Phys., 2017, 19, 11563-11570. 
13 (a) R. Wei, P. Song and A. Tong, J. Phys. Chem. C, 2013, 117, 3467-3474; (b) Z. Lin, X. Mei, E. Yang, X. Li, H. Yao, G. Wen, C.-T. Chien, T. J. Chow and Q. Ling, CrystEngComm, 2014, 16, 11018-11026; (c) S. Yagai, T. Seki, H. Aonuma, K. Kawaguchi, T. Karatsu, T. Okura, A. Sakon, H. Uekusa and H. Ito, Chem. Mater., 2016, 28, 234-241; (d) Y. Imai, T. Kinuta, K. Nagasaki, T. Harada, T. Sato, N. Tajima, Y. Sasaki, R. Kuroda and Y. Matsubara, CrystEngComm, 2009, 11, 1223-1226.

14 (a) P. S. Hariharan, D. Moon and S. P. Anthony, J. Mater. Chem. C, 2015, 3, 8381-8388; (b) L. Huang, Q. Liao, Q. Shi, H. Fu, J. Ma and J. Yao, J. Mater. Chem., 2010, 20, 159-166; (c) S. P. Anthony and S. M. Draper, J. Phys. Chem. C, 2010, 114, 11708-11716; (d) M. Abyan, D. de Caro and S. FeryForgues, Langmuir, 2009, 25, 1651-1658.

15 (a) Y. Hong, J. W. Y. Lam and B. Z. Tang, Chem. Commun., 2009, 4332-4353; (b) Yu. Hong, J. W. Y. Lam and B. Z. Tang, Chem. Soc. Rev., 2011, 40, 5361-5388; (c) D. Ding, K. Li, B. Liu and B. Z. Tang, Acc. Chem. Res., 2013, 46, 2441-2453; (d) J. Mei, N. L. C. Leung, R. T. K. Kwok, J. W. Y. Lam and B. Z. Tang, Chem. Rev., 2015, 115, 1171811940; (e) Q. Li and Z. Li, Adv. Sci., 2017, 4, 1600484.

16 (a) A. J. Cruz-Cabeza, S. M. Reutzel-Edens and J. Bernstein, Chem. Soc. Rev., 2015, 44, 8619-8635; (b) J.-P. Brog,
C.-L. Chanez, A. Crochet and K. M. Fromm, RSC Adv., 2013, 3, 16905-16931; (c) S. Varughese, J. Mater. Chem. C, 2014, 2, 3499-3516; (d) S. P. Anthony, ChemPlusChem, 2012, 77, 518-531; (e) D. Yan and D. G. Evans, Mater. Horiz., 2014, 1, 46-57; (f) J. Yang, Z. Ren, B. Chen, M. Fang, Z. Zhao, B. Z. Tang, Q. Peng and Z. Li, J. Mater. Chem. C, 2017, 5, 9242-9246.

17 T. Threlfall, Org. Process Res. Dev., 2003, 7, 1017-1027.

18 (a) A. Patra, N. Hebalkar, B. Sreedhar, M. Sarkar, A. Samanta and T. P. Radhakrishnan, Small, 2006, 2, 650-659; (b) C. G. Chandaluri and T. P. Radhakrishnan, Angew. Chem., Int. Ed., 2012, 51, 11849-11852; (c) S. P. Anthony and S. M. Draper, J. Phys. Chem. C, 2010, 114, 11708-11716; (d) M. R. Molla, D. Gehrig, L. Roy, V. Kamm, A. Paul, F. Laquai and S. Ghosh, Chem.-Eur. J., 2014, 20, 760-771; (e) A. Fernández-Mato, M. Svnchez-Andújar, B. PatoDoldán, M. A. Señarís-Rodríguez, C. Platas-Iglesias, D. Tordera, H. J. Bolink, J. M. Quintela, C. Peinador and M. D. García, Cryst. Growth Des., 2014, 14, 3849-3856.

19 C. Peebles, C. D. Wight and B. L. Iverson, J. Mater. Chem. C, 2015, 3, 12156-12163. 